\title{
Diabetes and Cancer Screening Rates among Appalachian and Non-Appalachian Residents of Kentucky
}

\author{
Steven T. Fleming, PhD, Margaret M. Love, PhD, and Keisa Bennett, MD, MPH
}

Background: Having diabetes may increase the odds of late-stage breast cancer. In Kentucky, the rates of late-stage disease are higher in rural than in urban areas, particularly in rural Appalachia. The objectives of the study were to examine the relationship between diabetes and cancer screening and to determine whether Appalachia residence modifies this association.

Methods: One thousand thirty Kentucky adults responded to a 2008 telephone survey that measured whether they had diabetes; lived in Appalachia; had guideline-concordant screening for breast (mammogram, clinical breast examination), cervical (Papanicolaou), and colorectal (fecal occult blood test or sigmoidoscopy/colonoscopy) cancer; and whether they reported receiving mammograms regularly every 1 to 2 years.

Results: Of the subjects, $16 \%$ had diabetes, $21 \%$ were Appalachian, and $32 \%$ were men. In multivariate analysis, women with diabetes had about half the odds of "regular" mammography screening (odds ratio, 0.56 ) compared with those without diabetes. Men and women in Appalachia had about half the odds of colonoscopy or sigmoidoscopy within the past 10 years (odds ratio, 0.54 ) compared with those living outside Appalachia.

Conclusions: Both having diabetes and living in Appalachia were negatively associated with current and regular cancer screening. Less screening may explain late-stage diagnosis among these populations. (J Am Board Fam Med 2011;24:682-692.)

Keywords: Appalachia, Cancer Screening, Diabetes

It is critical to understand the impact of illness on cancer screening because patients with undiagnosed cancer often have underlying chronic conditions that may influence screening decisions. In two earlier studies, Fleming and colleagues ${ }^{1,2}$ showed that some chronic conditions increase the risk of late- versus early-stage breast or prostate cancer, whereas others decrease that risk. Having diabetes, for example, increased the odds of late-stage breast

This article was externally peer reviewed.

Submitted 14 March 2011; revised 1 June 2011; accepted 6 June 2011.

From the Departments of Epidemiology (STF) and Family and Community Medicine (MML, KB), University of Kentucky, Lexington, KY.

Funding: This project was supported by grant no. R24 HS11845 from the Agency for Healthcare Research and Quality.

Conflict of interest: none declared.

Corresponding author: Steven T. Fleming, Department of Epidemiology, University of Kentucky, 121 Washington Avenue, Lexington, KY 40536 (E-mail: stflem2@uky.edu). cancer by approximately $20 \% .^{2}$ Fleming et $\mathrm{al}^{1,2}$ speculate a number of possible causes behind the equivocal relationship between chronic conditions and preventive care, including increased physician contact, competing demands on physician time, and a biomedical interaction among the different pathologies. Some of these pathways may be direct, such as when pharmacologic treatment of a chronic condition directly affects the risk of cancer, ${ }^{3}$ whereas others may be indirect, such as when a chronic condition leads to increased face time with a physician, more regular cancer screening, and a reduced risk of late-stage disease. Fleming et al's ${ }^{1,2}$ two studies, however, demonstrated that relationships between chronic conditions and stage of cancer remain even after controlling for the frequency of medical visits and cancer screening, which suggests that these factors alone cannot adequately explain the increased or decreased risk of a latestage cancer diagnosis. 
There is ample evidence that chronic conditions may influence patients' choice of screening. ${ }^{4,5}$ In addition, some studies have found that the accuracy of the screening tests themselves may be affected by the presence of chronic illness. Fenton et al ${ }^{6}$ for example, reported that the odds of a true positive screening (sensitivity of the test) is lower among those with increasing comorbidity burden, so, even if patients with multiple conditions receive screening, a cancer may not be detected. Therefore, the impact of chronic illness on screening may relate to physician practices, patient preferences, or test sensitivity.

The effect of diabetes on cancer screening may also depend on patient residence to the extent that barriers to accessible screening sites disproportionately affect rural residents, particularly those who live within the Appalachian region of the United States. According to Halverson and colleagues, ${ }^{7}$ the Appalachian region of the United States experiences an excess in premature mortality, ie, deaths before the age of 65 , for both breast and colorectal cancer. According to the Kentucky Breast Cancer Task Force, the incidence of late-stage breast cancer was significantly higher among Kentucky women than in the Surveillance, Epidemiology, and End Results (SEER) population (http://seer. cancer.gov)-Surveillance, Epidemiology, and End Results is a collection of US population-based cancer registries covering approximately $25 \%$ of the US population. Late stage breast cancer was higher in rural than in urban Kentucky and was highest of all in rural Appalachian Kentucky. ${ }^{8}$ Presumably, lower screening rates among rural and Appalachian residents result in proportionately less early-stage disease diagnoses and higher mortality rates, an assumption supported by Behavioral Risk factor Surveillance System (BRFSS) data demonstrating Appalachian screening rates for breast and cervical cancer approximately $3 \%$ below national levels. ${ }^{9}$ Considering the high rates of both diabetes and cancer in Kentucky as a whole and the Appalachian population in particular, we hypothesized that the pattern found by Fleming et $\mathrm{al}^{1,2}$ would also be applicable to people with and without diabetes in Kentucky. The purpose of this analysis was to examine the relationship between diabetes and cancer screening through a 2008 survey of 1030 Kentucky residents and to determine if residence in the Appalachian region of Kentucky is an effect modifier of this relationship.

\section{Methods}

\section{Procedures and Participants}

After obtaining institutional review board approval from the University of Kentucky, the University of Kentucky Survey Research Center conducted the survey during the time period of May 15 through June 30, 2008. Trained, experienced, and continuously monitored Survey Research Center interviewers conducted the interviews with households that were selected using a modified list-assisted, Waksberg-Mitofsky, random-digit dialing procedure for landline telephones. Up to 15 attempts were made for each telephone number in the sample, with up to 10 scheduled call backs if timing was "inconvenient." Eligible participants were adults, aged 18 years and older, who could complete the interview in English. The interviewer asked to speak with the adult in the household aged 18 or older who had most recently had a birthday.

The survey resulted in 1030 completed interviews, with an additional 201 subjects ruled ineligible. Subjects were ineligible if they were younger than 18 years of age or were from a household with no adults, could not speak English, were hearing impaired, or were not available for the entire period that the survey was in the field. Altogether, 2338 persons who were contacted refused to participate. With a total of 3559 calls and 1231 completed interviews or contacts ruled ineligible, we calculate a response rate of $34.6 \%$ according to the Council of American Survey Research Organization (http:// www.casro.org).

\section{Survey Items}

The entire survey consisted of five sets of questions: a first set of demographic questions such as age, sex, education, and race; a second and third set consisting of questions relating to asthma and diabetes, respectively; a fourth set of questions related to cervical, colorectal, and breast cancer screening; and a final set of questions that developed the concept of a "regular source of care" or "medical home." Survey items determined whether and when respondents had received each of the following cancer screening tests: fecal occult blood test (FOBT), colonoscopy or sigmoidoscopy, mammogram, clinical breast examination (CBE), and $\mathrm{Pa}-$ panicolaou test. Sigmoidoscopy and colonoscopy were combined into one item because of the possibility that respondents could not easily distin- 
guish them. In addition, women were asked whether they get mammograms regularly every 1 to 2 years. The survey items were drawn from the National Health Interview Survey ${ }^{10}$ and the Medical Expenditures Panel Survey, ${ }^{11}$ with the exception of time since CBE, which was based on similar items in the National Health Interview Survey and Medical Expenditures Panel Survey, and whether women regularly receive mammograms, which was a new item. It was intended to assess women's perception of a pattern of mammography screening over time that is not captured by the item about the most recent mammogram.

To capture screening at younger ages for future analyses, respondents were asked the cancer screening items if they were up to 10 years younger than the age recommended to begin screening as issued by the United States Preventive Services Task Force (USPSTF), ${ }^{12-15}$ the American Cancer Society, ${ }^{16-19}$ or the American College of Obstetricians and Gynecologists (ACOG) ${ }^{20}$ as of Spring 2008. The present analyses only included ages actually recommended for screening, not the younger ages. To gauge recency of screening, respondents could indicate "within the past year," "within the past 2 years," "within the past 5 years," or "5 or more years ago" for Papanicolaou, mammogram, CBE, and FOBT; and "within the past 10 years" or " 10 or more years ago" for sigmoidoscopy/colonoscopy.

\section{Analyses \\ Criteria for Inclusion in the Analyses and Currency of Cancer Screening}

Inclusion was based only on age and sex. The criteria for inclusion allow women and men to be 2 years older than the recommended beginning age for receiving screening (eg, 23 years old instead of 21 years old). Any testing met the criteria for screening, regardless of whether it was initiated for screening or for diagnosis. The criteria did not consider the results of previous tests.

Our criteria for whether respondents were eligible to receive screening and were current in their screening were based on the guidelines in place in Spring 2008. This was before the USPSTF updated its recommendations about breast cancer screening in late $2009^{21,22}$ and others about cervical cancer screening in 2009 and 2010. ${ }^{23,24}$ Specific cancer screening guidelines referenced are listed in the Appendix (available from the author upon request). ${ }^{12-20,25}$
Women 23 years of age and older were included in the cervical cancer screening analyses. They were considered current if they had received a $\mathrm{Pa}-$ panicolaou test during the past 2 years. For currency of screening, this criterion gives women an extra year in which to receive their Papanicolaou test compared with the 2008 recommendation from ACOG for annual screening. ${ }^{25} \mathrm{We}$ also analyzed the data using a period of 3 years to reflect newer guidelines and to allow for comparison to the BRFSS data. ${ }^{9}$ Women 42 years of age and older were included in the breast cancer screening analyses. They were considered current for any of the breast cancer screening tests if they had received the test during the past 2 years; this is the less conservative interval in the USPSTF and ACOG guidelines beginning with this age period. ${ }^{13,20} \mathrm{Men}$ and women 52 years of age and older were eligible for inclusion in the colorectal cancer screening analyses. For FOBT screening, patients were considered current if they were screened within 2 years, allowing an extra year compared with the USPSTF and American Cancer Society guidelines. ${ }^{14,15,19}$ For colonoscopy/sigmoidoscopy, women and men were considered current if they were screened within 10 years, the interval recommended for colonoscopy. ${ }^{14,15,19}$ Colonoscopy and sigmoidoscopy were combined because some patients may not be able to distinguish between them. They were not given an extra year to have received a colonoscopy or sigmoidoscopy because respondents are unlikely to distinguish 10 years from 11 years when recalling their screening compared with recalling the more recent tests within the past 2 years.

\section{Diabetes}

The survey attempted to distinguish between those who have diabetes, those with some type of prediabetic condition, and those without diabetes. Women were classified as diabetic based on answering "yes" to the question, Other than during pregnancy, have you ever been told by a doctor or health professional that you have diabetes or sugar diabetes? Men were classified as diabetic based on answering "yes" to the similar question, Have you ever been told by a doctor or health professional that you have diabetes or sugar diabetes? Both men and women were classified as "prediabetic" if they answered "yes" to the question, Have you ever been told by a doctor or other health professional that 
you have any of the following: prediabetes, impaired fasting glucose, impaired glucose tolerance, borderline diabetes, or high blood sugar?

\section{Appalachian Residents}

Appalachian residence within Kentucky was defined by county according to the Appalachian Regional Commission definitions. ${ }^{26}$ Fifty-four of Kentucky's 120 counties are in Appalachia.

\section{Statistical Analyses and Tests}

In addition to descriptive statistical analyses, we calculated unadjusted bivariate associations between each type of cancer screening and diabetes status; between each type of cancer screening and Appalachian versus non-Appalachian residence; and between each type of cancer and diabetes status stratified by Appalachian versus non-Appalachian residence. Note that diabetes status in this analysis excluded those diagnosed with prediabetes; however, a sensitivity analysis including prediabetic patients was performed. We also estimated multivariate logistic models for each screening test, controlled for age (in years), race (white/other), and education (less than high school, high school graduate or GED, some college). We tested for statistically significant differences in the odds ratios of stratified analyses using the Breslow-May test. All analyses used SAS software (Windows version 9.2, SAS, Inc., Cary, NC).

\section{Results}

Descriptive statistics for the 1030-person sample of Kentucky residents are reported in Table 1. The

Table 1. Descriptive Statistics of a Sample of 1030 Kentucky Residents, 2008

\begin{tabular}{|c|c|c|c|c|c|c|}
\hline \multirow[b]{2}{*}{ Strata } & \multicolumn{2}{|c|}{$\begin{array}{c}\text { Total } \\
(\mathrm{n}=1,030)\end{array}$} & \multicolumn{2}{|c|}{$\begin{array}{c}\text { Men } \\
(\mathrm{n}=332)\end{array}$} & \multicolumn{2}{|c|}{$\begin{array}{c}\text { Women } \\
(\mathrm{n}=698)\end{array}$} \\
\hline & Freq & Percent & Freq & Percent & Freq & Percent \\
\hline \multicolumn{7}{|l|}{ Age (years) } \\
\hline $0-24$ & 32 & 3.1 & 16 & 4.8 & 16 & 2.3 \\
\hline $25-49$ & 321 & 31.2 & 86 & 26.0 & 235 & 33.7 \\
\hline $50-64$ & 393 & 38.2 & 136 & 41.1 & 257 & 36.9 \\
\hline $65-74$ & 157 & 15.3 & 58 & 17.5 & 99 & 14.2 \\
\hline $75-85$ & 100 & 9.7 & 26 & 7.9 & 74 & 10.6 \\
\hline$\geq 85$ & 25 & 2.4 & 9 & 2.7 & 16 & 2.3 \\
\hline Appalachian region & & & & 19.3 & & \\
\hline Yes & 214 & 20.8 & 64 & 80.7 & 150 & 21.6 \\
\hline No & 813 & 79.2 & 268 & & 545 & 78.4 \\
\hline \multicolumn{7}{|c|}{ Married or living with partner } \\
\hline Yes & 643 & 63.1 & 232 & 70.1 & 411 & 59.7 \\
\hline No & 376 & 36.9 & 99 & 29.9 & 277 & 40.3 \\
\hline \multicolumn{7}{|l|}{ Race } \\
\hline White & 954 & 93.7 & 308 & 93.3 & 646 & 93.9 \\
\hline Black & 46 & 4.5 & 16 & 4.9 & 30 & 4.4 \\
\hline Asian & 4 & 0.4 & 1 & 0.3 & 3 & 0.4 \\
\hline Other & 14 & 1.4 & 5 & 1.5 & 9 & 1.3 \\
\hline Education & & & & & 98 & \\
\hline$<$ High school & 132 & 13.0 & 34 & 10.3 & 241 & 14.2 \\
\hline High school or GED & 347 & 34.1 & 106 & 32.1 & 349 & 35.0 \\
\hline College & 539 & 52.9 & 190 & 57.6 & & 50.7 \\
\hline \multicolumn{7}{|l|}{ Diabetes status } \\
\hline None & 813 & 79.1 & 256 & 77.1 & 557 & 80.0 \\
\hline Prediabetes & 52 & 5.1 & 14 & 4.2 & 38 & 5.5 \\
\hline Diabetes & 163 & 15.9 & 62 & 18.7 & 101 & 14.5 \\
\hline
\end{tabular}

Denominator for percents includes all data available for any given analysis (eg, $\mathrm{n}=1,027$ having Appalachian/non-Appalachian county data; $\mathrm{n}=1,028$ for diabetes status).

Freq, frequency; GED, General Educational Development. 
sample consists of approximately twice as many female as male respondents, with approximately $20 \%$ from the Appalachian region in Kentucky. The sample was mostly white and mostly married or living with a partner. Ninety percent of men and $86 \%$ of women had at least a high school education. We also report the prevalence of diabetes and prediabetes among the sample of 332 men and 698 women. Men have a somewhat higher prevalence of diabetes than women $(18.7 \%$ compared with $14.5 \%)$ but a somewhat lower level of prediabetes ( $4.2 \%$ vs $5.5 \%)$. We noted that our overall screening results were similar to BRFSS data from 2008 (http://www.cdc.gov/brfss/), including colon cancer screening, in which the prevalence of FOBT testing within the last 2 years in Kentucky was $17.2 \%$ and nationwide $21 \%$, and the prevalence of people who had ever had a colonoscopy or sigmoidoscopy was $63.7 \%$ in Kentucky and $62.2 \%$ nationwide.

In bivariate analyses (Table 2) we made six different comparisons between respondents with and without diabetes for the prevalence of screening examinations for three different cancer sites. Respondents with prediabetes were excluded from these analyses. Sensitivity analysis including prediabetes patients did not change any of the conclusions in terms of statistically significant results. Significantly fewer women with diabetes had had a mammogram during the past 2 years compared with those without diabetes (66.0\% vs $76.8 \% ; P=.03)$, and proportionately fewer participants reported regular mammography screening $(68.5 \%$ vs $81.6 \% ; P=.006)$. Having diabetes was not related to any type of colorectal cancer screening, nor did it seem to be related to $\mathrm{CBE}$. Because colorectal cancer was the only site analyzed that has screening recommendations for both women and men, it was also analyzed separately for each sex to disentangle possible gender effects. No relationship between diabetes and colorectal cancer screening was found for either men or women. The lower proportion of women with diabetes who reported cervical cancer screening within 2 years was only marginally significant $(66.7 \%$ vs $78.1 \% ; P<.06)$.

Table 2. Bivariate Associations between Diabetes Status* and Screening Examinations, Kentucky Residents, 2008

\begin{tabular}{|c|c|c|c|}
\hline & Frequency $^{\dagger}$ & Percent Screened & $P\left(\chi^{2}\right)$ \\
\hline \multicolumn{4}{|l|}{ Women, age $\geq 23$ years } \\
\hline \multicolumn{4}{|c|}{ Papanicolaou test within 2 years } \\
\hline With diabetes & 57 & 66.7 & \multirow[t]{2}{*}{.06} \\
\hline Without diabetes & 374 & 78.1 & \\
\hline \multicolumn{4}{|c|}{ Papanicolaou test within 3 years } \\
\hline With diabetes & 57 & 70.2 & \multirow[t]{2}{*}{.0007} \\
\hline Without diabetes & 374 & 84.3 & \\
\hline \multicolumn{4}{|l|}{ Women, age $\geq 42$ years } \\
\hline \multicolumn{4}{|l|}{ Regular mammography } \\
\hline With diabetes & 89 & 68.5 & \multirow[t]{2}{*}{.006} \\
\hline Without diabetes & 401 & 81.6 & \\
\hline \multicolumn{4}{|c|}{ Mammography within 2 years } \\
\hline With diabetes & 94 & 66.0 & \multirow[b]{2}{*}{.03} \\
\hline Without diabetes & 423 & 76.8 & \\
\hline \multicolumn{4}{|c|}{ Men and women, age $\geq 52$ years } \\
\hline \multicolumn{4}{|l|}{ FOBT within 2 years } \\
\hline With diabetes & 129 & 24.8 & \multirow[t]{2}{*}{.25} \\
\hline Without diabetes & 467 & 20.1 & \\
\hline \multicolumn{4}{|c|}{ Colonoscopy/ sigmoidoscopy within 10 years } \\
\hline With Diabetes & 130 & 63.1 & \multirow[t]{2}{*}{.72} \\
\hline Without Diabetes & 466 & 61.4 & \\
\hline
\end{tabular}

Bold values indicate $P \leq .05$.

*Patients with diabetes are subjects who have ever been told that they had diabetes by a health professional. Patients without diabetes are subjects who have not ever been told by a health professional that they had diabetes.

${ }^{\dagger}$ Frequency differs across strata because of age and sex eligibility and missing data.

FOBT, fecal occult blood test. 
When the data were analyzed for Papanicolaou testing within 3 years, however, the difference between diabetics and nondiabetics was clearly significant $(70.2 \%$ vs $78.1 \% ; P<.0007)$.

The bivariate analyses shown in Table 3 compare the prevalence of screening examinations for three different cancer sites among both men and women inside and outside the Appalachian region of Kentucky. Again, we excluded respondents with prediabetes from these analyses. Significantly fewer participants living in the Appalachian region of Kentucky indicated that they received "regular" mammography screening $(72.8 \%$ vs $81.7 \% ; P=.04)$. Proportionately more men and women in this region were concordant with guidelines for colorectal screening with an FOBT test within 2 years $(25.2 \%$ vs $19.8 \% ; P=$ $.01)$ but proportionately less for colonoscopy/ sigmoidoscopy within the last 10 years $(49.3 \%$ vs $67.0 \% ; P=.0007)$. Other associations between screening rates among Appalachian and non-Appalachian residents were not statistically significant.
The bivariate analyses shown in Table 4 also compared the prevalence of screening examinations for three different cancer sites among both men and women with and without diabetes. These analyses were stratified by whether the subjects lived in an Appalachian or non-Appalachian county of Kentucky. Within the non-Appalachian region, approximately 10\% less women with diabetes received regular mammograms compared with women without diabetes $(P=$ $.05)$; in contrast, these comparisons were not significant for Appalachian women. Appalachian residents with or without diabetes had substantially lower rates of colonoscopy/sigmoidoscopy within the last 10 years compared with those living in the non-Appalachian regions. We tested whether Appalachian residence was an effect modifier for diabetes and each of the screening modalities using a Breslow-May test; we found no statistically significant difference in odds ratios for each screening test.

As in the bivariate analyses, multivariate logistic models showed that having diabetes was neg-

Table 3. Bivariate Associations between Appalachian Residency and Screening Examinations, Kentucky Residents 2008

\begin{tabular}{|c|c|c|c|}
\hline & Frequency* & Percent Screened & $P\left(\chi^{2}\right)$ \\
\hline \multicolumn{4}{|c|}{ Women, age $\geq 23$ years with no hysterectomy } \\
\hline \multicolumn{4}{|c|}{ Papanicolaou test within 2 years } \\
\hline Appalachian & 108 & 70.5 & \multirow[t]{2}{*}{.11} \\
\hline Non-Appalachian & 344 & 77.9 & \\
\hline \multicolumn{4}{|l|}{ Pap test within 3 years } \\
\hline Appalachian & 108 & 75.9 & \multirow[t]{2}{*}{.11} \\
\hline Non-Appalachian & 344 & 84.6 & \\
\hline \multicolumn{4}{|l|}{ Women, age $\geq 42$ years } \\
\hline \multicolumn{4}{|l|}{ Regular mammograms } \\
\hline Appalachian & 114 & 72.8 & \multirow[t]{2}{*}{.04} \\
\hline Non-Appalachian & 405 & 81.7 & \\
\hline \multicolumn{4}{|c|}{ Mammography within 2 years } \\
\hline Appalachian & 122 & 73.0 & \multirow[t]{2}{*}{.57} \\
\hline Non-Appalachian & 427 & 75.9 & \\
\hline \multicolumn{4}{|c|}{ Men and women, age $\geq 52$ years } \\
\hline \multicolumn{4}{|l|}{ FOBT within 2 years } \\
\hline Appalachian & 135 & 25.2 & \multirow[t]{2}{*}{.01} \\
\hline Non-Appalachian & 494 & 19.8 & \\
\hline \multicolumn{4}{|c|}{ Colonoscopy/ sigmoidoscopy within 10 years } \\
\hline Appalachian & 136 & 49.3 & \multirow[t]{2}{*}{.0007} \\
\hline Non-Appalachian & 494 & 67.0 & \\
\hline
\end{tabular}

Bold values indicate $P \leq .05$.

*Frequency differs across strata because of age and sex eligibility requirements and missing data. FOBT, fecal occult blood testing. 
Table 4. Bivariate Associations between Diabetes Status* and Screening Examinations for Those in and outside of Appalachia, Kentucky Residents 2008

\begin{tabular}{|c|c|c|c|c|c|c|}
\hline \multirow[b]{2}{*}{ Screening Test } & \multicolumn{3}{|c|}{ Appalachian Resident } & \multicolumn{3}{|c|}{ Non-Appalachian Resident } \\
\hline & Frequency $^{\dagger}$ & $\%$ Screened & $P\left(\chi^{2}\right)$ & Frequency $^{\dagger}$ & \%Screened & $P\left(\chi^{2}\right)$ \\
\hline \multicolumn{7}{|l|}{ Women, age $\geq 23$ years } \\
\hline \multicolumn{7}{|c|}{ Papanicolaou test within 2 years } \\
\hline With diabetes & 15 & 60.0 & .34 & 42 & 69.1 & .11 \\
\hline Without diabetes & 90 & 72.2 & & 283 & 79.9 & \\
\hline \multicolumn{7}{|c|}{ Papanicolaou test within 3 years } \\
\hline With diabetes & 15 & 60.0 & .11 & 42 & 73.8 & .03 \\
\hline Without diabetes & 90 & 78.9 & & 283 & 86.6 & \\
\hline \multicolumn{7}{|l|}{ Women, age $\geq 42$ years } \\
\hline \multicolumn{7}{|c|}{ Regular mammography } \\
\hline With diabetes & 22 & 59.0 & .12 & 66 & 72.7 & .05 \\
\hline Without diabetes & 87 & 75.9 & & 313 & 83.1 & \\
\hline \multicolumn{7}{|c|}{ Mammography within 2 years } \\
\hline With diabetes & 23 & 65.2 & .37 & 70 & 67.1 & .07 \\
\hline Without diabetes & 94 & 74.5 & & 328 & 77.4 & \\
\hline \multicolumn{7}{|c|}{ Men and women, age $\geq 52$ years } \\
\hline \multicolumn{7}{|l|}{ FOBT within 2 years } \\
\hline With diabetes & 32 & 28.1 & .56 & 96 & 22.9 & .41 \\
\hline Without diabetes & 100 & 23.0 & & 366 & 19.1 & \\
\hline \multicolumn{7}{|c|}{ Colonoscopy/sigmoidoscopy within 10 years } \\
\hline With diabetes & 31 & 41.9 & .46 & 98 & 70.4 & .28 \\
\hline Without diabetes & 101 & 49.5 & & 364 & 64.6 & \\
\hline
\end{tabular}

Bold values indicate $P \leq .05$.

*Patients with diabetes are subjects who have ever been told by a health professional that they had diabetes. Patients without diabetes are subjects who have not ever been told by a health professional that they had diabetes.

${ }^{\dagger}$ Frequency differs across strata because of age and sex eligibility and missing data.

FOBT, fecal occult blood test.

atively associated with receiving regular mammograms and that Appalachian residence was negatively associated with having received a colonoscopy or sigmoidoscopy with the last 10 years (see Table 5). We tested whether Appalachian residence was an effect modifier for diabe-

Table 5. Multivariate Logistic Regression* of Six Screening Tests, Kentucky Residents 2008

\begin{tabular}{llc}
\hline Screening Test & Diabetes $^{\dagger}$ (yes/no) & Appalachian Resident (yes/no) \\
\hline Women, age $\geq 23$ years & & $0.78(0.45-1.34)$ \\
$\quad$ Papanicolaou test within 2 years & $0.89(0.46-1.74)$ & $0.66(0.36-1.22)$ \\
$\quad$ Papanicolaou test within 3 years & $0.62(0.30-1.26)$ & $0.71(0.43-1.19)$ \\
Women, age $\geq 42$ years & & $0.93(0.57-1.51)$ \\
$\quad$ Regular mammography & $\mathbf{0 . 5 6 ( 0 . 3 2 - 0 . 9 6 )}$ & $1.37(0.85-2.20)$ \\
Mammography within 2 years & $0.65(0.39-1.08)$ & $\mathbf{0 . 5 4}(\mathbf{0 . 3 6 - 0 . 8 0 )}$ \\
Men and women, age $\geq 52$ years & $1.44(0.90-2.33)$ & $1.24(0.81-1.91)$ \\
$\quad$ FOBT within 2 years &
\end{tabular}

Values shown as adjusted odds ratio $(95 \% \mathrm{CI})$. Bold values indicate $P \leq .05$.

${ }^{*}$ Controlling for education, race, and age.

${ }^{\dagger}$ Patients with diabetes are subjects who have ever been told by a health professional that they had diabetes. Patients without diabetes are subjects who have not ever been told by a health professional that they had diabetes.

FOBT, fecal occult blood test. 
tes and each of the screening modalities and found no significant effect modification.

\section{Discussion}

In this study we demonstrate clearly that, for Kentucky women, being up to date with breast cancer screening is negatively associated with having diabetes. Also, patients with diabetes had about half the odds of reporting "regular" mammography screening than those without diabetes. It is also noteworthy that Appalachian residents had about half the odds of reporting a colonoscopy/sigmoidoscopy within the last 10 years. Although such a cross-sectional study alone cannot demonstrate causality, our results suggest that having diabetes as a chronic illness presents a barrier to achieving guideline-concordant breast cancer mammography screening and that residents of Appalachia may face barriers to receiving colonoscopy/ sigmoidoscopy screening.

We also found that proportionately less Appalachian residents than non-Appalachian residents reported receiving "regular" mammograms, and proportionately fewer were up to date in their colorectal cancer screening with colonoscopy/sigmoidoscopy. However, proportionately more Appalachian residents than non-Appalachian residents were up to date with colorectal cancer FOBT screening. The diabetic-nondiabetic screening comparisons were significant for non-Appalachian but not Appalachian residents, but we did not confirm an effect modification of Appalachian residence on the relation between having diabetes and current screening with the Breslow-May test. These results should be interpreted cautiously because of the relatively small sample size of Appalachian residents. With this sample size, we should not conclude that Appalachian residence modifies the effect of diabetes on cancer screening.

Our results are consistent with findings of Schoen et $\mathrm{al}^{5}$ that physicians were less likely to request a mammogram for women with a chronic illness and with the Kiefe et $\mathrm{al}^{4}$ research showing that women with chronic illnesses were less likely to receive either mammography or CBE. It is unclear, however, why our results would single out a relationship between diabetes and breast cancer screening while showing only a marginal relationship for cervical cancer screening (significant in bivariate but not in regression analysis) and no association for colorectal cancer screening. There are several possible explanations.
First, the association between diabetes and obesity may play a role. This theory is consistent with studies by Ferrante et $\mathrm{a}^{27}$ indicating that obese women are less likely to adhere to mammography recommendations. It may be that obese women are more selfconscious in the situation of exposing their breasts to multiple strangers at a radiology suite, a hypothesis consistent with the research of Wee et $\mathrm{al}^{28}$ and Amy et al. ${ }^{29}$ In addition, mammography may be more technically difficult, painful, or unpleasant for obese women. Some studies support the hypothesis that mammograms produce more discomfort for women with particularly large or small breasts (see, for example, Papas and Klassen ${ }^{30}$ ). In our study, we did not know the extent to which such groups populate the diabetic and nondiabetic samples, and thus we could not infer whether or not discomfort mediated by breast size could have been a factor. However, we can speculate a smaller role for obesity to mediate rates of colorectal and cervical cancer screening because the discomfort posed by these tests is probably similar for normal-weight and moderately obese persons.

Second, a differential effect may be at play with breast cancer screening because of the level of provider involvement. It may seem counterintuitive that a recommendation for mammography, a screening generally well-accepted by women in the United States and that requires no office procedure, would be more affected by a chronic illness than those that require more office time (eg, Papanicolaou test) or extensive patient education (eg, colorectal cancer screening). However, the processes for mammography referral may be creating a different picture. Although colorectal cancer screening is initiated by the health care practitioner because of the need for counseling and referral, breast screening can be initiated and/or managed by the patient in conjunction with the screening facility. A small number of patients request their first mammogram themselves because of concerns raised through the media, public attention, or family/peer experience with breast cancer. ${ }^{31}$ Calls to the three major insurers in Kentucky revealed that only one of them requires a referral for screening mammograms. The other two allow for self-referral provided the patient's screening site is "in network." For a large number of Kentuckians, therefore, as long as the patient remains without symptoms or abnormalities on examination or imaging, subsequent screening appointments can be managed by the radiology provider and the patient (Humana, United Health Care, and Anthem Customer Service in Ken- 
tucky; telephone communications; April 2010). Similarly, the Kentucky Breast and Cervical Cancer Treatment Program (BCCTP), which funds free breast examination and mammography services for women without health insurance, requires these examinations to be conducted through local health departments and often do not involve the patient's primary care provider. ${ }^{32}$ Nevertheless, the literature is clear that the single most important factor in whether or not a woman receives a mammogram is whether it was recommended by her physician or provider. ${ }^{33}$ Though speculative, it may be that providers accustomed to the "automatic" nature of mammography and burdened by more complex visits with diabetic patients may be less likely to notice delays in mammography screening. Meanwhile, women with diabetes may be too overwhelmed by their physical condition, monitoring/medication regimen, or general stress of chronic illness to follow through reliably with mammography without their doctor's encouragement. Why the pattern for CBE generally matches that of mammography is more difficult to speculate, though the requirement to receive a CBE before mammography for those in the BCCTP population may explain some of this effect. Also, since 2002 the USPSTF has not made a recommendation for or against CBE because of "insufficient evidence" of benefit, and many providers no longer perform it routinely. ${ }^{22}$ The BCCTP program also provides $\mathrm{Pa}-$ panicolaou testing and follow-up for uninsured patients. Papanicolaou testing is less expensive and has fewer barriers than mammography, so many indigent patients continue to receive Papanicolaou tests at their primary care provider offices; however, the smaller number who presumably access all their gynecologic preventive care at the health department may also explain the relatively weaker relationship but definite trend between cervical screening and diabetes status.

Limitations of this study include the relatively small number of Appalachian residents that participated in this study. A larger sample might have provided the power to show other statistically significant relationships. Also, the inability to contact subjects without a landline telephone likely resulted in an undersampling of patients who are both younger and of lower socioeconomic status (SES). ${ }^{34}$ That the high school graduation rate of $87 \%$ in our study was significantly higher than the approximately $72 \%$ state average and the $60 \%$ to $65 \%$ average in Appalachian counties ${ }^{35}$ lends credence to the theory that low SES persons may have been undersampled. Although low SES is certainly associated with not having any telephone access, anecdotally, since the early 2000s there has been a movement away from landlines toward the use of only cell phones in both of the above demographic groups, which would raise the absolute effect of undersampling present as compared with older telephone-based studies. Even by 2006, more than $25 \%$ of adults younger than age 30 and $17 \%$ of adults in households earning $200 \%$ of the Federal poverty level or less were accessible only by cell phone. Households without a landline are also less likely to have health insurance or a usual source of care. ${ }^{34}$ Because all the screening tests in our study except for Papanicolaou tests concerned people older than 40 years of age, missing data from young cell phone-only users probably did not affect our results significantly. Unintentionally undersampling people with low SES means that fewer people with health insurance and a usual source of care would have responded. It is also important to note that Appalachian residents have disproportionately low SES. ${ }^{36}$ It is possible that the low number of Appalachian participants is partially due to our landline sampling technique and that some of the differences between Appalachian and non-Appalachian residents and between Appalachian diabetics and nondiabetics would have reached significance had we been able to sample cell phone-only households. It should be noted that both of these limitations actually bias the results to the null, so the significant findings in our study would likely be even stronger with more complete sampling. This could explain why differences between Appalachian and non-Appalachian populations were found in the Hall et $\mathrm{al}^{9}$ study using data from 1996 to 1998, before any significant cell phone penetration, whereas our data from 2007 to 2008 was not able to distinguish these differences.

Another limitation - and opportunity for future research-is that this survey did not enable us to assess the severity of diabetes in the sampled population or to assess multiple other chronic conditions. (The only other chronic condition surveyed was asthma, which will require an additional manuscript to address sufficiently.) Comorbid conditions such as hypertension, asthma, lipid disease, obesity, and other heart disease could have biased the results to the extent that these conditions either increase or decrease the likelihood of screening. The 2005 Fleming et $\mathrm{al}^{2}$ study showed that some conditions, such as diabetes, increased the risk of late-stage breast cancer, 
whereas others, such as cardiovascular disease, actually decreased the risk of late-stage disease. Thus, it is unclear whether multiple chronic conditions in the same person would cancel each other out (with regard to risk) or exacerbate the risk. Because it is also unclear how much chronic disease burden is present among diabetics compared with nondiabetics as a group, a reasoned assessment of the level and direction of bias cannot be done. Because colorectal cancer screening was relevant to both women and men, but breast cancer screening was measured only among women, we questioned whether the differences were because of different screening practices between men and women rather than between diabetics and nondiabetics. However, when men were excluded from the analysis, there was no difference in colorectal cancer screening between women with and without diabetes, just as there had been no difference with men and women combined.

It is worth noting that some of the recommendations for screening have changed since the initiation of this study. Papanicolaou tests, for instance, are not started before age 21 and are recommended at 3-year intervals after several normal Papanicolaou tests. ${ }^{23,24}$ The USPSTF has recently recommended against routine mammography for women younger than 50 years old. ${ }^{21,22}$ However, our results reflect the state of the guidelines at the time the survey was conducted.

Our results raise several issues for future study, chiefly the reasons for the difference between mammography and colorectal cancer screening with regard to effects of diabetes. Interventions focusing on provider prompts and reminders for screening may not be effective in cases in which screenings have become more automatic and less provider-directed. On the other hand, making providers aware of their role in encouraging screening for their diabetic patients could be an effective directed intervention if diabetics do indeed need assistance in keeping up with screenings. Regardless, it is important to better understand where to focus interventions, and a qualitative approach may shed light on the modern mammography experience for diabetics. Other possible research includes an analysis assessing other chronic conditions' and/or multiple comorbid conditions' effects on appropriate screening achievement, as well as which systems-based interventions could improve screening rates in patients with chronic illnesses.

\section{Conclusion}

Both having diabetes and living in Appalachia were negatively associated with current and regular cancer screening, suggesting that less screening could explain later-stage breast cancer among these populations. Several previous studies show a strong association between having diabetes mellitus and contracting cancer. There is ample evidence that this relationship is dependent on physiologic interactions, medication effects, and screening practices. Thus, the need for effective screening practices is even more relevant for diabetic patients than for patients without chronic illness and deserves further study and intervention.

The authors would like to thank Krista Mevoli, MPH, and Roshni Matnani, MPH, for their considerable assistance in developing the questionnaire.

\section{References}

1. Fleming ST, McDavid K, Pearce K, Pavlov D. Comorbidities and the risk of late-stage prostate cancer. Scientific World Journal 2006;6;2460-70.

2. Fleming ST, Pursley HG, Newman B, et al. Comorbidity as a predictor of stage of illness for patients with breast cancer. Med Care 2005;43(2): 132-40.

3. Harris AM, Warner BW, Wilson JM, et al. Effect of $\alpha 1$-adrenoceptor antagonist exposure on prostate cancer incidence: an observational cohort study. J Urol 2007;178:2176-80.

4. Kiefe CI, Funkhouser E, Fouad MN, May DS. Chronic disease as a barrier to breast and cervical cancer screening. J Gen Intern Med 1998;13(6): 357-65.

5. Schoen RE, Marcus M, Braham RL. Factors associated with the use of screening mammography in a primary care setting. J Community Health 1994; 19(4):239-52.

6. Fenton JJ, Barton MB, Geiger AM, et al. Screening clinical breast examination: how often does it miss lethal breast cancer? J Natl Cancer Inst Monogr 2005;35:67-71.

7. Halverson JA. An analysis of disparities in health status and access to heath care in the Appalachian region. November 2004. Available at: http://www. arc.gov/research/researchreportdetails.asp?REPORT_ $\mathrm{ID}=82$. Accessed 22 June 2010.

8. Friedell GH, Linville LH, Sorrell CL, Huang B. Kentucky breast cancer report card. J Ky Med Assoc 2003;101(10):449-54.

9. Hall HI, Uhler RJ, Coughlin SS, Miller DS. Breast and cervical cancer screening among Appalachian women. Cancer Epidemiol Biomarkers Prev 2002; 11(1):137-42. 
10. Centers for Disease Control and Prevention. National Health Interview Survey. 2009. Available at: http://www.cdc.gov/nchs/nhis.htm. Accessed 14 December 2009.

11. Agency for Healthcare Research and Quality. Medical Expenditure Panel Survey. Available at: http:// www.meps.ahrq.gov/mepsweb. Accessed 14 December 2009.

12. U.S. Preventive Services Task Force. Screening for cervical cancer. 2003. Available at: http://www. ahrq.gov/clinic/uspstf/uspscerv.htm. Accessed 22 June 2010.

13. U.S. Preventive Services Task Force. Screening for breast cancer: Recommendations and rationale. Ann Intern Med 2002;137(5 P 1):344-6.

14. U.S. Preventive Services Task Force. Screening for colorectal cancer: recommendation and rationale. Ann Intern Med 2002;137(2):129-31.

15. U.S. Preventive Services Task Force. Screening for colorectal cancer. 2008 Available at: http://www. ahrq.gov/clinic/uspstf/uspscolo.htm. Accessed 22 June 2010.

16. Saslow D, Runowicz CD, Solomon D, et al. American Cancer Society guideline for the early detection of cervical neoplasia and cancer. J Low Genit Tract Dis 2003;7(2):67-86.

17. Smith RA, Saslow D, Sawyer KA, et al. American Cancer Society guidelines for breast cancer screening: update 2003. CA Cancer J Clin 2003; 53(3):141-69.

18. Warner E, Yaffe M, Andrews KS, et al. American Cancer Society guidelines for breast screening with MRI as an adjunct to mammography. CA Cancer J Clin 2007;57(2):75-89.

19. Agency for Healthcare Research and Quality. National Guideline Clearinghouse. Guideline summary: screening and surveillance for the early detection of colorectal cancer and adenomatous polyps, 2008: a joint guideline from the American Cancer Society, the US MultiSociety Task Force on Colorectal Cancer, and the American College of Radiology. 2008. Available at: http://www.guidelines.gov/summary/summary.aspx? doc_id=14346\&nbr=7214. Accessed 22 June 2010.

20. Agency for Healthcare Research and Quality. National Guidelines Clearinghouse. Guideline summary: breast cancer screening. 2003. Available at: http://www. guidelines.gov/content.aspx? $\mathrm{id}=34275$ \&search $=$ breast+cancer+screening. Accessed 22 June 2010.

21. U.S. Preventive Services Task Force. Screening for breast cancer: U.S. Preventive Services Task Force recommendation statement. Ann Intern Med 2009; 151(10):716-26, W-236.

22. U.S. Preventive Services Task Force. Screening for breast cancer. 2009. Available at: http://www.ahrq. gov/clinic/uspstf/uspsbrca.htm. Accessed 22 June 2010.

23. American Cancer Society. American Cancer Society guidelines for the early prevention of cancer. June 2011. Available at: http://www.cancer.org/docroot/
PED/content/PED_2_3X_ACS_Cancer_Detection_ Guidelines_36.asp? sitearea=PED Accessed 22 June 2010.

24. Davis AJ, Journal Watch. Major changes in ACOG cervical cytology screening recommendations. Available at: http://womens-health.jwatch.org/cgi/ content/full/2009/1123/1. Accessed 22 September 2011.

25. Agency for Healthcare Research and Quality. National Guideline Clearinghouse. Guideline summary: cervical cytology screening. Available at: http://www. guidelines.gov/summary/summary.aspx?doc_id= $15274 \&$ nbr $=007506 \&$ string $=$ cervical + AND + cancer + AND+screening. Accessed 28 June 2010.

26. Appalachian Regional Commission. Counties in Appalachia. Available at: http://www.arc.gov/index.do? nodeId=27 Accessed 14 December 2009.

27. Ferrante JM, Chen PH, Crabtree BF, Wartenberg D. Cancer screening in women: body mass index and adherence to physician recommendations. Am J Prev Med 2007;32(6):525-31.

28. Wee CC, McCarthy EP, Davis RB, Phillips RS. Obesity and breast cancer screening. J Gen Int Med 2004;19:324-31.

29. Amy NK, Aalborg A, Lyons P, Keranen L. Barriers to routing gynecological cancer screening for white and African-American obese women. Int $\mathrm{J}$ Obes (London) 2006;30:147-55.

30. Papas MA, Klassen AC. Pain and discomfort associated with mammography among urban low-income African-American women. J Community Health 2005;30(4):253-67.

31. Reynolds HE, Jackson VP. Self-referred mammography patients: analysis of patients' characteristics. AJR Am J Roentgenol 1991;157:481-4.

32. Kentucky Cabinet for Health and Family Services. Breast and cervical cancer treatment program (BCCTP). Available at: http://chfs.ky.gov/dms/bcctp.htm. Accessed 28 September 2011.

33. Meissner HI, Breen N, Taubman ML, Vernon SW, Graubard BI. Which women aren't getting mammograms and why? (United States). Cancer Causes Control. 2007;18:61-70.

34. Blumberg SJ, Luke JV. Coverage bias in traditional telephone surveys of low-income and young adults. Public Opin Q 2007;71(5):734-49.

35. The Foundation for a Healthy Kentucky. High school graduation (percent adults age 25 or older), 2005-2009. Available at: http://kentuckyhealthfacts. org/data/topic/show.aspx?ind=2. Accessed 24 June 2010.

36. Mary Babb Randolph Cancer Center. Underlying socioeconomic factors influencing health disparities in the Appalachian region. 2008. Available via Appalachian Regional Commission website at: http://www.arc.gov/ research/ResearchReports.asp?F_Category $=7$. Accessed June 22, 2010. 